Our Nature (2007)5: 75-80

\title{
Avian diversity during rehabilitation stage of Chimdi Lake, Sunsari, Nepal
}

\author{
R.Surana ${ }^{1}$, B.R Subba and K.P Limbu \\ P.G Campus,Biratnagar, Nepal \\ ${ }^{1}$ Email:ranjanasurana@yahoo.com
}

Received: 28.10.2007, Accepted: 10.12.2007

\begin{abstract}
Avian diversity of Chimdi Lake during rehabilitation stage was studied. 109 species of birds belonging to 34 families were recorded. Maximum 64 species were recorded in March 2004 and 20 species recorded in July 2004.Out of total; bird species $33.94 \%$ were migratory, $25.68 \%$ were resident, $24.77 \%$ were winter visitors and $15.96 \%$ were summer visitors. On the basis of abundance, $41.28 \%$ were scarce, $22.9 \%$ were occasional, $21.1 \%$ were fairly common and $14.6 \%$ were common. The Lake area was found to be rich in avian diversity although the Lake was not fully rehabilitated.

Key words: Avian diversity, Chimdi Lake, , seasonal status
\end{abstract}

\section{Introduction}

The information regarding the avian diversity during rehabilitation stage in any lake area is very few. The present study has attempted to know both present and past condition of the lake in order to have an idea about the restoration of the lake again. If the lake is restored again it can provide habitat for many important species of bird. Nepal being rich in avian diversity, the study will help to add a new spot for bird sighting. Heinen (1987) studied the birds of Koshi Tappu wildlife reserve and Koshi Barage in Eastern Nepal. Subba (1994,1995 and 1997) made checklist of bird of Dharan, Biratnagar and Gajurmukhi VDC,Ilam respectively. Gori et.al.,(2003) studied the re-colonization of water bird following the wetland and rehabitation (Hortobagy National Park, Hungary). Singh and Roy (1990) studied the systematics of birds colonizing Kawar Lake (Begusarai, Bihar). The lake has attracted the attention of conservationists, so the present study explored the status of the lake and made specific suggestions to be included in planning of the lake for its restoration.

\section{Study area}

Chimdi Lake, locally known as 'Birju Tal' is the study area located at Chimdi village development committee (VDC), ward no. 3 and 4, Sunsari district, Nepal. It is about 12 $\mathrm{km}$ west from Nepal's main industrial city Biratnagar. Its geographical coordinates are $87^{\circ} 10^{\prime} 51.3^{\prime \prime}$ E longitude and $26^{\circ} 29^{\prime} 23.5^{\prime \prime} \mathrm{N}$ latitude. It is situated at an elevation of $70 \mathrm{~m}$ above mean sea level. The total area of the lake is approximately 101.6 hectare.

\section{Materials and methods}

Study of Avifauna of Chimdi Lake area was carried out from December 2003 to February 2005. Monthly census of bird was 
Ranjana Surana, B.R. Subba and K.P.Limbu / Our Nature (2007)5: 75-80

done in order to know their population and status. The population of birds was estimated from 6 am to 9 am by direct counting method. Birds were observed within transect of $200 \mathrm{~m}$. Binocular, digital camera, and field books were used during bird observation. The field books of Ali and Ripley (1986), Fleming et al. (2000) and Shrestha (2000) were used in the field for bird identification.

\section{Results}

On the basis of field observation 109 species of birds belonging to 34 families were recorded (Table1). Out of total species, 28 species were resident, 27 species were winter visitor arriving the lake area during winter (September to November) and leaving the area by summer (late April), 17 species were summer visitor arriving the lake area during summer (March to June) and departing on the commencement of winter (September, November). 37 species were migratory birds passing through lake area on their way to and from nesting grounds (Table 1). On the basis of census made at the lake area and its surrounding, abundance of the bird species was given (Table 1). 16 species were common, 23 species were fairly common, 25 species were occasional and 45 species were scarce.

Total numbers of individuals counted during the study period were 3675 (Table 1). Variation of bird species and total number of individuals of birds species on monthly basis have been shown in (Figure 1 and 2) respectively. Seasonal status and abundance of bird on percentage basis have been depicted in Figure 3 and 4 respectively.

\section{Discussion}

The biodiversity of the Nepalese fauna is revealed by birds for of the total bird species found throughout the entire area of South Asia, half are found along the foot hills of the Nepal Himalayas (Shrestha, 2000). The change in vegetation composition in relation to the stage of successional development is often determining factor on the types and abundance of bird inhabiting the area (Bhattarai, 1988).

Butterworth et al., (2003) studied water bird and water chemistry relations in shallow wetland basins in the Western Boreal Forest (WBF) which is the second most important waterfowl habitat in North America and concluded that understanding the relation between water birds and productivity is a crucial component of conservation planning. Chimdi Lake also supported thousands of water fowls but dramatic changes resulted due to anthropogenic influences. The lake is now at restorative phase.

In the present study 109 species of birds belonging to 34 families were recorded. On December 2003, 48 species birds were seen which declined to 39 species in December 2004 , within a period of one year a fall in number of species was recorded and this may be attributed to unsustainable use of lake thus destroying the birds' habitat. Maximum number of species recorded during study period of 15 months was 64 in the month of March 2004. This may be due to late departure of winter visitors and early arrival of summer visitors. Least number of birds' species (20) was recorded in July 2004 and this may be due to local migration of resident bird departure of winter visitors and commencement of monsoon season. However decreasing trend in species number was observed and this may be due to destruction of bird's habitat.

On the basis of abundance $41.28 \%$ birds were scarce and seen rarely, $22.9 \%$ were occasional, $21.1 \%$ were fairly common and $14.6 \%$ were common birds of Chimdi Lake 


\section{Ranjana Surana, B .R. Subba and K .P.L imbu / O ur N ature (2007)5: 75-80}

Table1 A vian Diversity of Chimdi Lake area and its surroundings

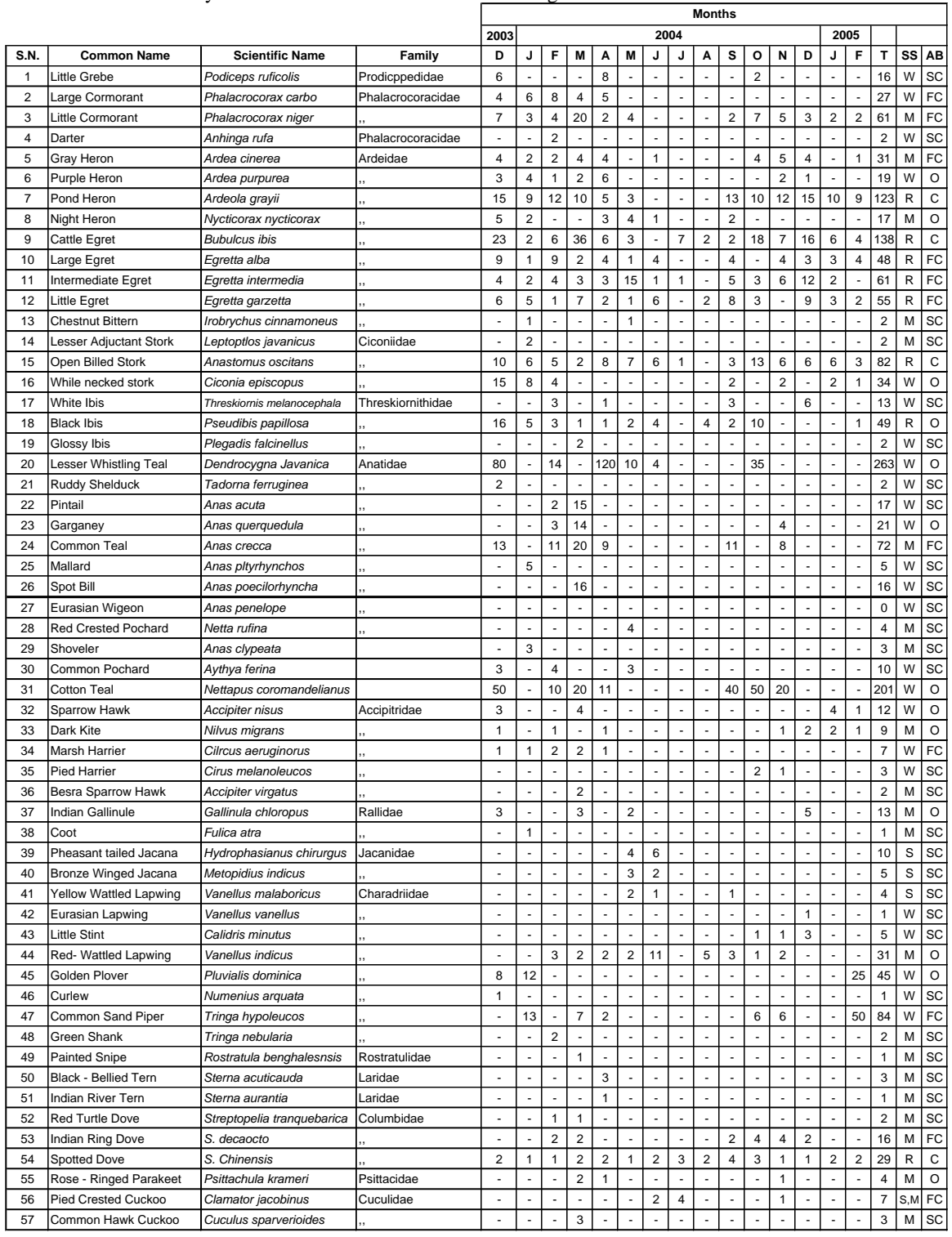




\section{Ranjana Surana, B .R. Subba and K .P.L imbu / O ur N ature (2007)5: 75-80}

\begin{tabular}{|c|c|c|c|c|c|c|c|c|c|c|c|c|c|c|c|c|c|c|c|c|c|}
\hline 58 & Eurasian Cuckoo & Cuculus canorus &, & - & - & - & - & 2 & - & - & - & - & - & - & - & - & - & - & 2 & $\mathrm{M}$ & $\mathrm{SC}$ \\
\hline 59 & Indian Cuckoo & Cuculus micropterus &,. & - & - & 1 & 2 & - & - & 2 & - & - & - & - & - & - & - & - & 5 & $\mathrm{~s}$ & $\mathrm{sC}$ \\
\hline 60 & Koel Cuckoo & Eudynamyus scolopocea &., & - & - & 3 & 2 & 2 & - & - & - & - & - & - & - & - & 3 & 7 & 17 & $\mathrm{~s}$ & $\mathrm{FC}$ \\
\hline 61 & Large Coucal & Centropus sincnsis &, & 1 & 1 & 3 & 1 & - & - & - & 1 & 2 & 4 & - & 2 & 2 & - & 1 & 18 & $\mathrm{R}$ & $\mathrm{FC}$ \\
\hline 62 & Small Pied King fisher & Ceryle rudis & Alcedinidae & 4 & 1 & 4 & 4 & 1 & 2 & 3 & 1 & 1 & 2 & 1 & 1 & 2 & 2 & 1 & 30 & $\mathrm{R}$ & $\mathrm{FC}$ \\
\hline 63 & Blue Eared King Fisher & Alcedo meninting &, & 1 & 1 & - & 2 & - & - & $=$ & - & - & 1 & - & - & - & - & - & 5 & $\mathrm{M}$ & $\mathrm{SC}$ \\
\hline 64 & Eurasian King fisher & Alcedo atthis & ,. & 1 & 2 & - & - & - & - & - & - & - & 3 & - & 1 & - & 2 & 1 & 10 & $\mathrm{M}$ & $\mathrm{FC}$ \\
\hline 65 & White breasted Kingfisher & Halcyon smyrnensis & & 2 & 1 & - & - & - & 2 & $=$ & - & 1 & - & 2 & 2 & - & 3 & 2 & \begin{tabular}{|c|c|}
15 \\
\end{tabular} & $\mathrm{M}$ & $\mathrm{FC}$ \\
\hline 66 & Blue tailed Bee eater & Merops philippinus & Meropideae & - & - & 2 & - & - & - & - & - & - & - & - & - & - & - & - & 2 & $\mathrm{M}$ & 0 \\
\hline 67 & Green Bea - Eater & Merops orientalis & & $=$ & - & - & 1 & - & - & 1 & - & - & - & - & - & - & - & - & 2 & $\mathrm{M}$ & $\mathrm{SC}$ \\
\hline 68 & Crimson breasted Barbet & Megalaima haemacephala & Capitonidae & - & - & - & 2 & - & - & - & 2 & - & - & - & - & - & - & -- & 4 & $\mathrm{M}$ & $\mathrm{SC}$ \\
\hline 69 & Blue throated Barbet & Megalaima asicatica & Capitondiae & - & - & 3 & - & - & - & 2 & - & - & - & - & - & - & - & - & 5 & $\mathrm{M}$ & 0 \\
\hline 70 & Ashy crowned finch lark & Eremopterix grisea & Alaudidae & 3 & 3 & 2 & 4 & 4 & 3 & 4 & - & - & - & 2 & 2 & 3 & 8 & 2 & \begin{tabular}{|l|l}
40 & 1 \\
\end{tabular} & $\mathrm{RM}$ & $\mathrm{C}$ \\
\hline 71 & Short toed Lark & Calandrella cinerea & ., & 7 & 5 & 3 & 3 & 4 & 3 & 2 & - & - & - & - & 3 & 5 & - & - & 35 & $\mathrm{RM}$ & $\mathrm{C}$ \\
\hline 72 & Crested Lark & Galerida cristata &., & - & - & $-\cdot$ & - & - & 1 & - & - & - & - & $-\cdot$ & - & - & \begin{tabular}{|l|l}
20 \\
\end{tabular} & 2 & \begin{tabular}{|l|l|l|l|}
23 & 1 \\
\end{tabular} & $\mathrm{RM}$ & 0 \\
\hline 73 & Bush Lark & Mirafra assamica & ,. & 2 & 1 & 7 & - & - & 3 & 3 & - & - & - & - & - & 10 & 3 & 2 & \begin{tabular}{|l|l}
31 \\
\end{tabular} & $\mathrm{R}, \mathrm{M}$ & $\mathrm{FC}$ \\
\hline 74 & Sand Martin & Riparia paludicola & Hirundinidae & - & 13 & - & 6 & 20 & - & - & 2 & - & - & - & - & - & 2 & - & 43 & $\mathrm{~s}$ & $\mathrm{FC}$ \\
\hline 75 & Barn Swallow & Hirundo rustica &., & - & 15 & - & - & - & - & - & - & - & - & - & - & 3 & - & - & \begin{tabular}{|l|l}
18 \\
\end{tabular} & $\mathrm{M}$ & $\mathrm{sC}$ \\
\hline 76 & Straited Swallow & Hirundo daurica & ., & - & - & - & - & - & - & - & - & - & - & - & - & 6 & - & - & 6 & $M$ & $\mathrm{SC}$ \\
\hline 77 & Brown Shrike & Lanius cristatus & Laniidae & - & - & - & - & - & - & - & - & - & - & 2 & 2 & - & - & - & 4 & $\mathrm{M}$ & $\mathrm{sc}$ \\
\hline 78 & Black Naped Oriole & Oriolus chinensis & Oriolidae & - & 2 & 2 & 3 & 2 & 2 & - & - & - & - & - & - & - & - & - & 11 & $\mathrm{~s}$ & 0 \\
\hline 79 & Black Headed Oriole & Oriolus xanthornus & ., & - & 2 & 1 & 1 & - & - & - & - & - & - & - & - & - & - & - & 4 & $\mathrm{~s}$ & 0 \\
\hline 80 & Golden Oriole & Oriolus orilous & ., & - & - & 3 & 1 & 1 & - & - & - & - & - & - & - & - & - & - & 5 & $\mathrm{~s}$ & 0 \\
\hline 81 & Ashy Drongo & Dicrurus lucophaeus & Dicruridae & 10 & - & - & 3 & 2 & 6 & - & - & 2 & 49 & 10 & 12 & 6 & - & 12 & 112 & $\mathrm{R}$ & C \\
\hline 82 & Black Drongo & Dicrurus adsimilis & ," & 19 & 7 & 14 & 76 & 26 & 17 & 38 & 20 & 22 & 16 & 25 & 18 & 4 & 17 & \begin{tabular}{l|l}
10 & 3 \\
\end{tabular} & 329 & $\mathrm{R}$ & $\mathrm{C}$ \\
\hline 83 & Gray headed Myna & Sturnus melabaricus & Sturnidae & 3 & - & - & 2 & - & - & - & - & - & - & - & - & - & - & - & 5 & $\mathrm{M}$ & 0 \\
\hline 84 & Pied Myna & Sturnus contra &., & 12 & 9 & 11 & 7 & 3 & 7 & 13 & 9 & 8 & 5 & 25 & 11 & 2 & \begin{tabular}{|l|l|}
16 \\
\end{tabular} & \begin{tabular}{|l|l|}
12 & 1 \\
\end{tabular} & 150 & $\mathrm{R}$ & C \\
\hline 85 & Common Myna & Acridotheres tristis & ,. & 23 & 16 & 33 & 14 & \begin{tabular}{|l|l|l|}
22 \\
\end{tabular} & 12 & 16 & 18 & 12 & 14 & 30 & 12 & 4 & 25 & $21212 r$ & 272 & $\mathrm{R}$ & C \\
\hline 86 & Bank Myna & Acridotheres giginianus &., & 4 & 4 & 3 & 6 & 9 & 9 & 7 & 3 & 2 & 7 & 12 & 8 & - & - & 3 & 77 & $\mathrm{R}$ & $\mathrm{C}$ \\
\hline 87 & Jungle Myna & Acndotheres fuscus & Sturnidae & - & - & - & - & - & 1 & - & - & - & - & 4 & - & - & - & - & 5 & $\mathrm{M}$ & 0 \\
\hline 88 & Indian Tree Pie & Dendrocitta vagabunda & Corvidae & - & - & 2 & 2 & - & - & - & - & 1 & 1 & 2 & 2 & 2 & - & 1 & 13 & $\mathrm{R}$ & $\mathrm{FC}$ \\
\hline 89 & House Crow & Corvus splendens &., & 6 & 9 & 4 & 11 & 7 & 6 & 8 & 5 & 9 & 7 & 16 & 10 & 7 & 14 & 11 & 130 & $\mathrm{R}$ & $\mathrm{C}$ \\
\hline 90 & Jungle Crow & Corvus macrorhynchos &., & 4 & 3 & 5 & 2 & 3 & 4 & 4 & 3 & 4 & 6 & 2 & 5 & 3 & 2 & 4 & 54 & $\mathrm{R}$ & $\mathrm{C}$ \\
\hline 91 & Dark Cuckoo Shrike & Coracina melaschistas & Campephagidae & - & - & - & 2 & - & - & - & - & - & - & - & - & - & - & 1 & 3 & $\mathrm{M}$ & 0 \\
\hline 92 & Red Whiskered Bulbul & Pycnonotus jocosus & Pycnonotiadae & 2 & 1 & 3 & 6 & 5 & 3 & 2 & 3 & 1 & 3 & 1 & 3 & 4 & 2 & 2 & 41 & $\mathrm{R}$ & $\mathrm{C}$ \\
\hline 93 & Red Vented Bulbul & Pycnonotus cafer & ., & 3 & 6 & 2 & 5 & 3 & 7 & - & - & 3 & - & - & 9 & - & 6 & - & 44 & $\mathrm{R}$ & C \\
\hline 94 & Jungle Babbler & Turdoides striatus & Timaliidae & 3 & 10 & 6 & 2 & 2 & 7 & - & - & - & - & - & 5 & - & 4 & - & 39 & $\mathrm{R}$ & $\mathrm{FC}$ \\
\hline 95 & Paradise Fly Catcher & Terpsiphone paradisi & Muscicapidae & - & - & - & - & - & 1 & - & - & - & - & - & - & - & - & - & 1 & $\mathrm{~s}$ & $\mathrm{SC}$ \\
\hline 96 & Gray headed flycatcher & Culicicapa ceylonensis & Muscicapidae & - & 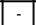 & - & - & - & 2 & - & - & - & - & - & - & - & - & - & 2 & $\mathrm{M}$ & $\mathrm{sC}$ \\
\hline 97 & Robin Dayal & Copsychus saularis & Turdidae & - & 3 & 5 & - & - & - & 2 & 2 & 3 & 8 & 4 & 3 & 4 & - & - & 34 & $\mathrm{R}$ & $\mathrm{FC}$ \\
\hline 98 & Eurasian Black Bird & Turdus merula & ,", & - & - & - & 1 & - & - & - & - & - & - & - & - & - & - & - & 1 & $\mathrm{~s}$ & $\mathrm{SC}$ \\
\hline 99 & Black Red Start & Phoenicurus ochruros & ., & - & - & - & 3 & - & - & - & - & - & - & - & - & 2 & - & - & 5 & $\mathrm{~s}$ & $\mathrm{sC}$ \\
\hline 100 & Collard Bush Chat & Saxicola torquata & ,. & - & - & - & 3 & - & - & - & - & - & - & 1 & - & 2 & - & - & 6 & $\mathrm{~s}$ & $\mathrm{SC}$ \\
\hline 101 & Pied Wagtail & Motacilla alba & Motacillidae & 4 & - & - & 7 & - & - & - & - & - & 3 & 6 & 7 & 12 & - & 3 & 42 & WM & $\mathrm{FC}$ \\
\hline 102 & Yellow Headed Wagtail & Mitacilla citreda & Motacillidae & - & 6 & 1 & 3 & - & - & - & - & - & - & - & 3 & 8 & - & - & 21 & w & 0 \\
\hline 103 & Gray Wagtail & Motacilla caspica & & - & - & 3 & - & - & - & - & - & - & - & 2 & - & 12 & - & 6 & 23 & w & 0 \\
\hline 104 & Paddy Field Pipit & Anthus nova eseelandiae & & - & 2 & 2 & 4 & - & - & - & 6 & 2 & - & 2 & 3 & 3 & - & 3 & \begin{tabular}{|l|l|l|l|}
27 \\
\end{tabular} & RM & $\mathrm{FC}$ \\
\hline 105 & Spotted Munia & Lonchura punctulata & Ploceidae & - & - & - & - & - & - & - & 10 & - & 16 & - & - & - & - & - & 26 & $\mathrm{~s}$ & 0 \\
\hline 106 & House Sparrow & Passer domesticus &., & 17 & 20 & 7 & 13 & 10 & 6 & 14 & 9 & 12 & 21 & \begin{tabular}{|l|l|l|l|}
23 \\
\end{tabular} & 14 & 12 & 18 & 172 & 213 & $\mathrm{R}$ & C \\
\hline 107 & Crested Bunting & Melophus lathami & Emberizidae & - & - & - & - & - & - & - & - & - & 3 & 2 & - & - & - & - & 5 & $\mathrm{M}$ & $\mathrm{SC}$ \\
\hline 108 & Tailor Bird & Orthotomus sutorius & Sylviidae & 6 & - & - & 4 & - & - & - & - & - & - & - & - & - & - & - & 10 & $\mathrm{~s}$ & $\mathrm{sC}$ \\
\hline \multirow[t]{8}{*}{109} & Yellow throated leaf warbler & Phylloscopus cantator &, & - & - & - & 6 & 1 & - & - & - & - & - & 7 & - & - & - & - & 14 & $\mathrm{~s}$ & $\mathrm{SC}$ \\
\hline & & & Total & 431 & 237 & 255 & 423 & 340 & \begin{tabular}{|l|}
176 \\
\end{tabular} & 172 & \begin{tabular}{|l|l|}
110 \\
\end{tabular} & 100 & \begin{tabular}{|l|l|}
276 \\
\end{tabular} & \begin{tabular}{|l|l|}
353 \\
\end{tabular} & 237 & \begin{tabular}{|l|}
207 \\
\end{tabular} & 189 & 230 & & & \\
\hline & & & No. of Species & 48 & 47 & \begin{tabular}{|l|l|}
54 & \\
\end{tabular} & \begin{tabular}{l|l|}
64 & \\
\end{tabular} & \begin{tabular}{|l|l}
45 & \\
\end{tabular} & \begin{tabular}{|l|l|}
40 & \\
\end{tabular} & \begin{tabular}{l|l|}
30 & \\
\end{tabular} & \begin{tabular}{|l|l|}
20 & \\
\end{tabular} & \begin{tabular}{|l|l|}
21 \\
\end{tabular} & \begin{tabular}{|l|l|}
35 & \\
\end{tabular} & \begin{tabular}{|l|l|}
39 & \\
\end{tabular} & 44 & \begin{tabular}{|l|l|}
39 & \\
\end{tabular} & \begin{tabular}{|l|l|}
28 \\
\end{tabular} & 36 & & & \\
\hline & SS $=$ Seasonal Status & $A B=$ Abundance & & & & & & & & & & & & & & & & & & & \\
\hline & $\mathrm{R}=$ Resident & $\mathrm{C}=$ Common & & & & & & & & & & & & & & & & & & & \\
\hline & W = Winter Visitor & FC = Fairly Common & & & & & & & & & & & & & & & & & & & \\
\hline & $\mathrm{S}=$ Summer Visitor & $\mathrm{O}=$ Occasional & & & & & & & & & & & & & & & & & & & \\
\hline & $\mathrm{M}=$ Migrant & $\mathrm{SC}=$ Scarce & & & & & & & & & & & & & & & & & & & \\
\hline
\end{tabular}


Ranjana Surana, B .R. Subba and K .P.Limbu / O ur N ature (2007)5: 75-80

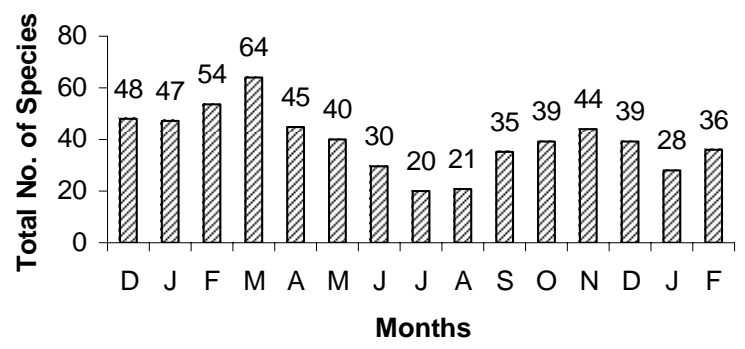

Figure $1 \mathrm{~V}$ ariation of bird species on monthly basis.

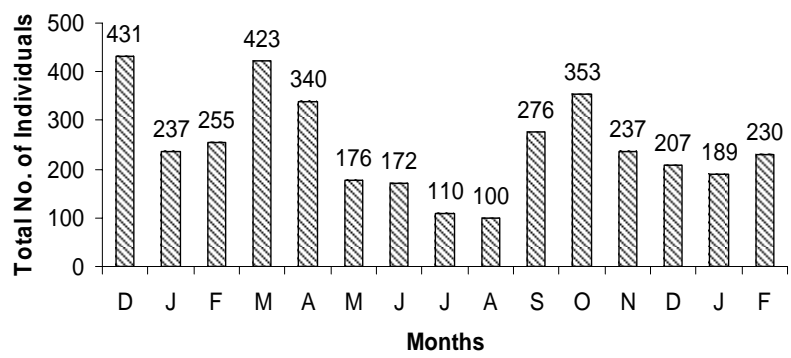

Figure $2 \mathrm{~V}$ ariation of total number of individuals of bird species on monthly basis.

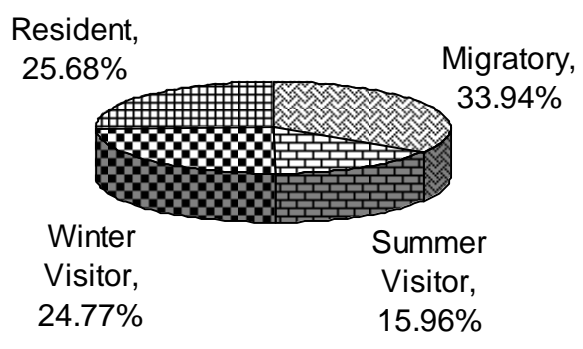

Figure 3. Seasonal Status of birds on percentage basis

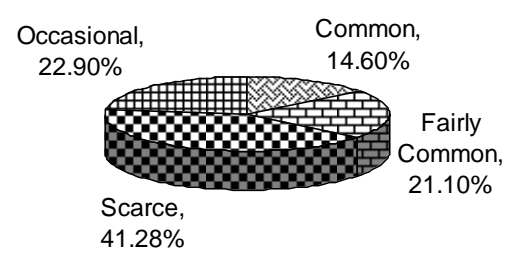

Figure4 A bundance of birds on percentage basis 
Ranjana Surana, B.R. Subba and K.P.Limbu / Our Nature (2007)5: 75-80

and its surrounding area. $25.68 \%$ of birds were resident, $33.94 \%$ were migrant $24.77 \%$ were winter visitors and $15.96 \%$ were summer visitors of the Chimdi Lake and its surrounding area.

In Koshi Tappu Wildlife Reserve 461 species of birds representing 58 families are recorded (Baral, 2000). At least 176 species breed in the reserve and 180 species are passage migrant or visitors (IUCN, 1998). The Chimdi Lake area accounts $23.64 \%$ and $58.62 \%$ of total birds of Koshi Tappu Wildlife Reserve in terms of number of species and family respectively. Similarly Chimdi Lake area represents $20.76 \%$ of total birds of Chitwan National Park in terms of number of species. Shrestha (2000) reported 525 species of birds in Chitwan National Park. Subba (1994) recorded 96 species of birds in Biratnagar whereas Chimdi Lake area and its surrounding show greater number of species than those recorded in Biratnagar.

Lake area shows rich diversity of avifauna. It can play important role in harbouring the birds that visit Koshi Tappu Wildlife Reserve and can be developed as a bird sanctuary parallel to Koshi Tappu Wildlife Reserve if restoration of the lake will be done in the time to come.

\section{Acknowledgements}

We are thankful to the local people w ho helped during field visits. We are thankful to the Department of Zoology, P.G Campus (T.U), Biratnagar for the laboratory facilities. The first author is thankful to Pro Public and NAST for financial support.

\section{References}

Ali, S and S. D. Ripley 1986. Handbook of the Birds of India and Pakistan, Vol. V, Oxford University Press.

Baral, H.S. 2000. Birds of Koshi. Bird Conservation Nepal, Pub. No. 3, DNPWC, Kathamndu, Nepal.

Bhattarai, K. 1988. A Comparative Study on Habitat Ecology of Cavity Nesting Birds in Isle (Disturbed) and Icharni (Protected) Along Riverine Forest of Chitwan National Park, Central Department of Zoology T. U. Kathmandu, Nepal. (M.Sc. Dissertation)

Butterworth, E. G. Ducks Stewart, K. Devito and W. Bell 2003. Water birds and water chemistry relations in shallow wetland basins in the Western Boreal Forest. 4th Conference Aquatic Birds Working Group of Societas International in Limnologia (SIL).

Fleming R. L (Sr.), R. L. Fleming (Jr.) and L.S. Bangdel 2000. Birds of Nepal with reference to Kashmir and Sikkim. First Adarsh Impression, Gaurav Offset, Delhi.

Gori S.Z., G. Lakatos, C.S. Aradi and S. Andrikovics 2003. Re-colonization of water birds following the wetland rehabilitation (Hortobagy N. P., Hungary). $4^{\text {th }}$ Conference, Aquatic Birds Working Group of Societas Internationalism Limnologia (SIL)

Heinen, J. T. 1987. New Seasonal Range Records and Status Updates for Birds in Koshi Tappu Wildlife Reserve and Koshi Barrage, Nepal, J. Nat. Hist. Mus., Vol. 11 No. $(1-4)$ : $41-50$.

IUCN 1998. An interpretation and education system for koshi Tappu wildlife reserve and its Buffer zones. IUCN Nepal.

Shrestha, T.K. 2001. Herpetology of Nepal, A study of Amphibians and Reptiles of Trans Himalayan Region of Nepal, India, Pakistan and Bhutan, Variety Printers, Kathmandu, Nepal.

Singh, J. P. and S. P. Roy 1990. Some Aspects of Ecology of Birds of Kawar Lake, Begusarai (Bihar), J. Fresh Water Biol. 2 (3):75 - 188.

Subba, B. R. 1994. Checklist of Birds of Biratnagar, Newsletter for Bird Watchers, 34 (6): 128 - 129.

Subba, B.R. 1995. Checklist of Birds of Dharan, Newsletter for bird watchers 35 (3): 50-53.

Subba, B.R.1997.Checklist of Birds of Gajurmukhi V.D.C.of Ilam.Vishleshan Vol.2: 20-23. 